\title{
Cluster expansions for fermion fields by the time dependent Hamiltonian approach
}

\author{
David C. Brydges* \\ Department of Mathematics, The University of Michigan, Ann Arbor, Michigan 48104
}

(Received 3 Novernber 1975)

A cluster expansion is given for a fermion field moving in an external field according to the interaction $\Psi \psi \phi$

in one space dimension.

\begin{abstract}
The purpose of this paper is pedagogic: to demonstrate, in a problem relatively untrammelled by technical complications, how a cluster expansion may be developed for the Yukawa model in one space dimension. The cluster expansion is based on the time dependent Hamiltonian approach devised by Federbush. ${ }^{1}$ It appears to the author that the method of integrating out all the fermions ${ }^{2-4}$ being developed by McBryan, Seiler, and Simon should be capable of producing a more streamlined version of the cluster expansion than the one implied by this paper. However, since it may be slightly surprising that the cluster expansion is not tied to a manifestly covariant approach, and, furthermore, the same general idea has the applications ${ }^{1}$ in statistical mechanics, perhaps this paper is not devoid of interest.
\end{abstract}

Federbush's idea is to replace the use of covariances with Dirichlet conditions in Ref. 5 by Hamiltonians for which regions are isolated by potential barriers. The extremes wherein two regions are completely isolated or in full communication are interpolated by varying the heights of these barriers. It will be seen that barriers parallel to the spatial axis may be incorporated by making the Hamiltonians suitably time dependent. The main disadvantage of this approach is that the heights of the barriers get involved in the estimates. The other technical complication which will occur in all approaches to a cluster expansion for the Yukawa model is the nonpositivity (in any sense) of the propagator for fermions. The positivity properties of the free Euclidean boson covariance were a useful aid in Ref. 5 .

The formal structure of the expansion is the same (except that integrations over interpolating variables run from zero to infinity) as in Ref. 5, [see Eqs. (3.13)]. This will become evident in Sec. 1. Therefore, rather than repeating much of the material in Ref. 5 , this paper is confined to establishing suitable analogs to the key ingredients of the convergence proof in Ref. 5. These are the formula for differentiating the measure (1.7) in Ref. 5 (this is discussed in Sec. 2), and Proposition (5.3) in Ref. 5, which is the subject of Secs. 3-5. The technical complications mentioned above are all buried in Sec. 5. The main point is the identity (1.11), which relates part of the free fermion propagator to Brownian motion. A "hand-wave" at the full Yukawa theory in one space dimension is given in the Appendix.

The external field model discussed here is almost as singular as the Yukawa model (the vacuum energies of both are logarithmically divergent), hence the cluster expansion is given for fields with a momentum cutoff and the convergence is established uniformly in this cutoff. The difference between the two models may be expressed in the following way: The external field $\phi=\phi(x)$ in this paper is assumed to belong to $L^{3}(\mathbb{R})$ locally uniformly. If $\phi$ were a boson field, this condition would be logarithmically divergent in a momentum cutoff. Needless to say, this extra divergence entails considerable complications for the Yukawa model. Nevertheless, the author feels that the interesting parts of a cluster expansion (by this method) for the Yukawa model are contained in this paper.

\section{NOTATION}

With the same notation and representation as in Ref. 6 the fermion field $\psi$ at time zero is given by

$$
\begin{aligned}
\psi(x)= & (4 \pi)^{-1 / 2} \int \exp (i p x) \\
& \times\left\{v(p) b^{\prime *}(p)+u(-p) b(-p)\right\} \omega^{-1 / 2}(p) d p,
\end{aligned}
$$

where the spinors are

$$
u(p)=\left[\begin{array}{c}
{[\omega(p)-p]^{1 / 2}} \\
-[\omega(p)+p]^{1 / 2}
\end{array}\right], \quad v(p)=\left[\begin{array}{c}
{[\omega(p)-p]^{1 / 2}} \\
{[\omega(p)+p]^{1 / 2}}
\end{array}\right] .
$$

The cluster expansion will be developed by devising a family of time dependent Hamiltonians which will interpolate between the usual one and one which does not propagate across or into any of the shaded regions in Fig. 1. Each shaded region is centered on a line of integral ordinate. The width $\epsilon$ of the "barriers" will be chosen below. To this end, the free Hamiltonian is modified as follows: The single particle kinetic energy is given by the operator $\left(M_{0}^{2}-\Delta\right)^{1 / 2}$, where $\Delta=d^{2} / d x^{2}$. Define the $(\tau, s)$ dependent operator,

$$
\omega(\tau, s)=\left(M_{0}^{2}-\Delta+\sum_{b} s_{b} \chi_{b}\right)^{1 / 2},
$$

where $\chi_{b}=\chi_{b}(x, \tau)$ is a characteristic function of one of the shaded regions in Fig. 1 , which particular one being specified by the subscript $b$, and $s=\left(s_{b}\right)$ is a multivariable (each $s_{b} \geqslant 0$ ) parametrizing the interpolation. By a well-known theorem, $\omega^{2}(s, \tau)$ converges pointwise in $\tau$ in the sense of strong convergence of the resolvent, as a given $s_{b} \rightarrow \infty$, to the analogous operator with Dirichlet conditions on $b$. (A proof can easily be constructed using the path space representation.) This implies, by functional analysis, that $\exp [-\omega(\tau, s)]$ converges strongly, pointwise in $\tau$. Define the corresponding time dependent free-field Hamiltonian by 


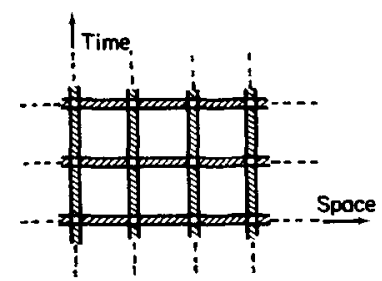

FIG. 1. Illustration of an infinite array of barriers partitioning $\mathbb{R}^{2}$.

second quantization of $\omega(\tau, s)$; i. e. ,

$H_{\mathrm{OF}}(\tau, s)=\int d x d y \omega(\tau, s, x, y)\left[b^{*}(x) b(y)+b^{\prime *}(x) b^{\prime}(y)\right]$,

where $\omega(\tau, s, x, y)$ is the distribution kernel of the operator (1.3).

The interpolation $(1.4)$ is not sufficient to make different squares independent because $\phi$ contains the nonlocal operators $\omega^{-1 / 2}, u, v$. Also it is necessary to use a cutoff on the momentum which likewise is not local. Choose a positive function $\kappa=\kappa(x)$ in $C^{\infty}(\mathbb{R})$ with support in the interval $\left(-\frac{1}{10}, \frac{1}{10}\right)$, normalized so that $\int \kappa(x) d x=1$. Define $\kappa_{\varepsilon}(x)=(1 / \epsilon) \kappa(x / \epsilon)$ where $\epsilon>0$. At the outset of the cluster expansion, choose some $\epsilon>0$ and cut off all fields by the replacement $\psi(x) \rightarrow\left(\kappa_{\epsilon} * \psi\right)(x)$. The nonlocal operators in $\kappa_{\epsilon} * \psi$ are the factors $\hat{\kappa}_{\epsilon}(p) \omega^{-1 / 2}(p) u(p)$ and $\hat{\kappa}_{\epsilon}(p) \omega^{-1 / 2}(p) v(p)$. Let $f(\tau, s, x, y)=\Pi_{b \in \Gamma(\tau, x, y)}\left[1 /\left(1+s_{b}\right)\right]$ where $\Gamma(\tau, x, y)$ is the set of barriers $b$ that intersect the straight line joining $(x, \tau)$ and $(y, \tau)$. Now let

$$
\begin{aligned}
& \begin{array}{l}
w_{+}(x, y, \tau, s, \epsilon) \\
\quad=(4 \pi)^{-1 / 2}\left(\hat{\kappa}_{\epsilon} \omega^{-1 / 2} u\right)^{-}(x-y) f(\tau, s, x, y), \\
w_{-}(x, y, \tau, s, \epsilon) \\
\quad=(4 \pi)^{-1 / 2}\left(\hat{\kappa}_{\epsilon} \omega^{-1 / 2} v\right)^{-}(x-y) f(\tau, s, x, y),
\end{array}
\end{aligned}
$$

and define

$$
\begin{aligned}
\psi(x, \tau, s, \epsilon)= & \int d y\left[w_{+}(x, y, \tau, s, \epsilon) b(y)\right. \\
& \left.+w_{-}(y, x, \tau, s, \epsilon) b^{*}(y)\right] .
\end{aligned}
$$

Note that $\psi(x, \tau, 0, \epsilon)=\left(\kappa_{\epsilon} * \psi\right)(x) ; s=0$ means $s_{b}=0$ for all $b$; at $s=\infty, w_{ \pm}(x, y)=0$, whenever $x, y$ are separated by a barrier or whenever $x$ or $y$ is in a barrier. From now on, dependences on $s, \tau, \epsilon$ will frequently be suppressed. Corresponding to these interpolated fields, define the propagator, letting $x=\left(x^{\prime}, t\right), y=\left(y^{\prime}, u\right) \in \mathbb{R}^{2}$, by

$$
S(x, y, s)=T\left\langle\bar{\psi}\left(x^{\prime}, t, s\right) \exp \left[-\int_{-\infty}^{\infty} H_{\mathrm{O} F}(\tau, s) d \tau\right] \psi\left(y^{\prime}, u, s\right)\right\rangle,
$$

where $T$ is the time ordering operator. All time dependences are piecewise constant, so (1.7) is easily defined. More explicitly, the propagator is given by

$S(x, y, s)$

$$
=\left\{\begin{array}{l}
\int d x_{1}^{\prime} d y_{1}^{\prime} \bar{w}_{-}\left(x^{\prime}, x_{1}^{\prime}, t, s\right) p\left(x_{1}^{\prime}, t, y_{1}^{\prime}, u, s\right) \\
\quad \times w_{-}\left(y_{1}^{\prime}, y^{\prime}, u, s\right), \quad t>u, \\
-\int d x_{1}^{\prime} d y_{1}^{\prime} w_{+}\left(y^{\prime}, y_{1}^{\prime}, u, s\right) p\left(y_{1}^{\prime}, u, x_{1}^{\prime}, t, s\right) \\
\quad x_{w_{+}}\left(x_{1}^{\prime}, x^{\prime}, t, s\right), \quad u>t,
\end{array}\right.
$$

where $p\left(x^{\prime}, t, y^{\prime}, u\right)$ is the kernel of the operator defined by the time ordered exponential

$$
T \exp \left(-\int_{\min (t, u)}^{\max (t, u)} \omega(\tau, s) d \tau\right) .
$$

At $s=0, S$ reduces to the usual propagator. At $s=\infty$, all unshaded squares in Fig. 1 become independent. The proof is left to the reader.

The kernel $p\left(x^{\prime}, t, y^{\prime}, u\right)$ is a positive function and can be related to Brownian motion, which will be essential for estimates. The principle whereby this is obtained in probability theory is called subordination. Within the context of semigroups it can be obtained by: $t>0, \beta>0$,

$$
\begin{aligned}
\exp (-t \beta)= & (1 / i \pi) \int_{-\infty}^{\infty} d k_{0} k_{0}\left(k_{0}^{2}+\beta^{2}\right)^{-1} \exp \left(i k_{0} t\right) \\
= & \int_{0}^{\infty} d s\left((1 / i \pi) \int_{-\infty}^{\infty} d k_{0} k_{0} \exp \left(-k_{0}^{2} s\right) \exp \left(i k_{0} t\right)\right) \\
& \times \exp \left(-s \beta^{2}\right) \\
= & \int_{0}^{\infty} d s t^{-2} \mu\left(s / t^{2}\right) \exp \left(-s \beta^{2}\right)
\end{aligned}
$$

where $\mu(s)=(1 / 2 \sqrt{\pi}) \exp (-1 / 4 s) s^{-3 / 2}$. The essential point is that $\mu$ is positive. Hence the kernel of $\exp [-t \omega(\tau, s)]$ at fixed $(\tau, s)$ may be expressed in terms of the kernel of $\exp \left[-t \omega^{2}(\tau, s)\right]$ which is positive by virtue of its relation to Brownian motion. The operator $(1.9)$ is a product of operators with the form $\exp [-t \omega(\tau, s)]$.

The family of interacting Hamiltonians will now be defined. They are time dependent, but at $s=0$ they reduce to the normal time independent cutoff Hamiltonian for a fermion field interacting with an external field $\phi$.

$$
\begin{aligned}
H(\tau, s, \Lambda)= & H_{\mathrm{OF}}(\tau, s)+\int_{\Lambda_{\tau}} d x \phi(x) \\
& \times: \Psi(x, \tau, s, \epsilon) \psi(x, \tau, s, \epsilon): d x+E_{\epsilon}(\Lambda)
\end{aligned}
$$

where $\Lambda \subset \mathbb{R}^{2}$, is a bounded, measurable set and $\Lambda_{\tau}$ denotes its spatial cross-section at time $\tau$. The external field $\phi$ is assumed to satisfy, for all $x_{1} \in \mathbb{R}$,

$$
\left[\int_{x_{1}}^{H x_{1}}|\phi(x)|^{3} d x\right]^{1 / 3} \leqslant \text { const } .
$$

The vacuum energy renormalization is inspired by perturbation theory:

$$
\begin{aligned}
E_{\epsilon}(\Lambda)= & (4 \pi)^{-1} \int d p_{1} d p_{2} \mid\left(\chi_{\Lambda} \phi\right)^{\wedge}\left(p_{1}+p_{2}\right)^{2} \frac{\omega_{1} \omega_{2}-p_{1} p_{2}-M_{0}^{2}}{\omega_{1} \omega_{2}} \\
& \times \frac{1}{\omega_{1}+\omega_{2}}\left[\hat{\kappa}^{2}\left(\epsilon p_{1}\right) \hat{\kappa}^{2}\left(\epsilon p_{2}\right)(2 \pi)^{2}\right]
\end{aligned}
$$

where $\chi_{\Lambda}=\chi_{\Lambda}(x, \tau)$ is the characteristic function of $\Lambda$, and - denotes the fourier transform with respect to $x$ only. Hence $E_{\epsilon}(\Lambda)$ is a function of $\tau$.

From Fig. 1 it will be seen that $\mathbb{R}^{2}$ has been partitioned into the following subsets: large squares (unshaded), small squares (unshaded), and rectangles (shaded). Let $\nabla$ denote an element of this partition. The projection of this partition onto the spatial axis yields a partition of the latter into long and short intervals. Let $I$ label an element in this partition. Let $J$ label the 
elements of the similarly obtained partition of the time axis.

The barriers, i. e., shaded rectangles in Fig. 1, will be chosen of width $\epsilon$. Those barriers whose short dimension is in the space direction are called "space barriers;" the others are "time barriers." The subset of $\mathbb{I R}^{2}, \Lambda$ in (1.12) is restricted to be a union of $\nabla \mathrm{s}$ so that (1.12) has a stepwise time dependence and time orderings are easily defined.

The cluster expansion is used to analyze quantities of the form

$$
\int d x W(x) T\left\langle\prod_{i=1}^{p} \psi \#\left(x_{i}\right) \exp \left[-\int H(\tau, \Lambda) d \tau\right]\right\rangle,
$$

where $x=x_{1}, x_{2}, \ldots, x_{p}$ with $x_{i} \in \mathbb{R}^{2}, x_{i}=\left(x_{i}^{\prime}, t_{i}\right)$, $W \in L^{2}\left(\mathbb{R}^{2 p}\right), \psi_{\#}^{\#}=\psi$ or $\psi$. Generally, at the beginning of the expansion, $s=0$ and $\psi, H$ are the usual fields and Hamiltonian obtained by setting $s=0$ in (1.6) and (1.4). In this case the ${ }^{\#}$ s will have no time dependence; however, the $t_{i}$ s and $\tau$ must still be left in (1.15) as "dummy" variables because of their other role, time ordering the expression. The expansion is obtained by replacing $\psi \#$ s and $H$ by their $s$ dependent analogs and using the fundamental theorem of calculus together with factorizations and resummations exactly as in Ref. 5 . The proof that (1.15) factors in the appropriate manner along contours where $s=0$ can be carried out by first approximating the exponential by a polynomial, whereupon the factorization is an immediate consequence of Wick's theorem.

\section{THE ANALOG TO THE FORMULA FOR DIFFERENTIATING THE MEASURE IN REF. 5}

As essential ingredient in the convergence proof of the cluster expansion in Ref. 5 is its formula (1.7) for differentiating the measure. A similar formula also holds in this framework, namely

$$
\begin{aligned}
\frac{d}{d s_{b}} & T\left\langle P(\bar{\psi}(s), \psi(s)) \exp \left[-\int H(\tau, s, \Lambda) d \tau\right]\right\rangle \\
& =T\left\langle\left(S^{\prime} \Delta_{\bar{\psi}}\right) P(\bar{\psi}(s), \psi(s)) \exp \left[-\int H(\tau, s, \Lambda) d \tau\right]\right\rangle,
\end{aligned}
$$

where $P$ is a polynomial and

$$
\left(S ! \Delta_{\tilde{\psi} \psi}\right)=-\int\left(\frac{d}{d s_{b}} S(x, y, s)\right) \frac{\delta}{\delta \bar{\psi}(x)} \frac{\delta}{\delta \psi(y)} d x d y
$$

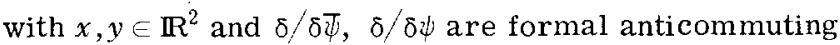
functional derivatives. The proof of $(2.1)$ is very easy in the special case where $\phi=0$ so that $H(\tau, s, \Lambda)$ $=H_{\mathrm{OF}}(\tau, s)$, for then the expectation on the left of (2.1) may be evaluated by using the anticommutation rules and the identity

$$
\begin{aligned}
& b(x) \exp \left[-\lambda H_{\mathrm{OF}}(s, \tau)\right] \\
& \quad=\exp \left[-\lambda H_{\mathrm{OF}}(s, \tau)\right](\exp [-\lambda \omega(s, \tau)] b)(x),
\end{aligned}
$$

together with similar ones for $b^{*}, b^{\prime}, b^{\prime *}$. This special case can be used to approximate the case when $\phi \neq 0$.

\section{THE EXPONENT}

In the next section, the following estimate will be used to control the exponential part of (1.15). For $M_{0}$ sufficiently large,

$$
H(\tau, s, \Lambda) \geqslant- \text { const }\left|\Lambda_{\tau}\right|
$$

uniformly in $\epsilon, \tau$, and $s$, where $\left|\Lambda_{\tau}\right|$ is the length of $\Lambda_{\tau} \subset \mathbb{R}$. The $s$ dependence in the $H_{\mathrm{OF}}(s)$ is bounded below by $H_{O F}(s) \geqslant H_{O F}(0)$. This is implied by the operator estimate $\omega(s) \geqslant \omega(0)$ which is implied by $\omega^{2}(s) \geqslant \omega^{2}(0)$ because operator estimates remain valid on taking square roots. The last bound is obvious. Thus (3.1) is implied by

$H_{\mathrm{OF}}+\int_{\Lambda_{\tau}} \phi(x): \bar{\psi}(x, \tau, s, \epsilon) \psi(x, \tau, s, \epsilon): d x+E_{\epsilon}(\Lambda)$

$\geqslant-$ const $\left|\Lambda_{\tau}\right|$.

The pure creation and annihilation parts, $V_{p}$, of the interaction have the form

$$
\begin{aligned}
& \int d p_{1} d p_{2}\left[v\left(p_{1}, p_{2}\right) b^{*}\left(p_{1}\right) b^{r *}\left(p_{2}\right)\right. \\
& \left.-\bar{v}\left(p_{1}, p_{2}\right) b\left(p_{1}\right) b^{\prime}\left(p_{2}\right)\right]
\end{aligned}
$$

for some $\epsilon, \tau, s, \phi$ dependent function $v$. The operator $H_{\mathrm{OF}}+V_{p}+E_{\epsilon}(\Lambda)$ will now be bounded below by a firstorder dressing transformation originally due to Glimm. ${ }^{7}$ In this situation, it is possible to use a particularly simple form of this transformation. ${ }^{6,8}$ First construct an operator $\Gamma V_{p}$ to satisfy

$$
\left[H_{\mathrm{OF}}, \Gamma V_{p}\right]=V_{p} \text {. }
$$

Note that (3.4) requires $\Gamma V_{p}$ be antisymmetric. Thus let

$$
\begin{aligned}
\Gamma V_{p}= & \left(\int d p_{1} d p_{2} v\left(p_{1}, p_{2}\right) \frac{1}{\omega\left(p_{1}\right)+\omega\left(p_{2}\right)}\right. \\
& \left.\times b^{*}\left(p_{1}\right) b^{*}\left(p_{2}\right)\right)-(\text { its adjoint }) .
\end{aligned}
$$

Define dressed operators by

$$
\begin{aligned}
& \tilde{b}(p)=b(p)+\left[b(p), \Gamma V_{p}\right], \\
& \tilde{b}^{\prime}(p)=b^{\prime}(p)+\left[b^{\prime}(p), \Gamma V_{p}\right] .
\end{aligned}
$$

Obtain an inequality by calculating the positive operator $\int d p \omega(p)\left[\tilde{b}^{*}(p) \tilde{b}(p)+\tilde{b}^{\prime *}(p) \tilde{b}^{\prime}(p)\right]$. Thus

$$
\begin{aligned}
\tilde{b}^{*}(p) \tilde{b}(p)= & b^{*}(p) b(p)+\left[b^{*}(p) b(p), \Gamma V_{p}\right] \\
& +\left[b^{*}(p), \Gamma V_{p}\right]\left[b(p), \Gamma V_{p}\right] .
\end{aligned}
$$

Normal order the operators in the last term of (3.7). The normal ordered form is a negative operator because of the anticommutation relations. Hence,

$$
H_{\mathrm{OF}}+V_{p}+\int d p_{1} d p_{2}\left|v\left(p_{1}, p_{2}\right)\right|^{2}\left(\omega_{1}+\omega_{2}\right)^{-1} \geq 0 .
$$

The third term corresponds to the contraction during the normal ordering. It diverges as $\epsilon \rightarrow 0$, however, up to a term which is greater than the right-hand side of (3.2), it cancels with $E_{\epsilon}(\Lambda)$. This calculation is not very difficult, using some of the devices developed in Sec. 5 .

By repeating the above argument with $\int d p \tilde{\omega}\left[\tilde{b}^{*} \tilde{b}\right.$ $\left.+\widetilde{b}^{\prime *} \tilde{b}^{\prime}\right]$ where $\tilde{\omega}=\omega-c \omega^{\tau^{\prime}}$ with $\tau^{\prime}<1$ and $c$ chosen so 
that $\tilde{\omega}>0$, the result can be strengthened to

$$
H_{\mathrm{OF}}+V_{p}+E_{\epsilon}(\Lambda) \geqslant c N_{\tau^{\prime}}-\text { const }\left|\Lambda_{\tau}\right|,
$$

where $N_{\uparrow}$ is the second quantization of $\omega^{\tau^{\prime}}$. For $M_{0}$ sufficiently large the remaining part of the interaction, $V_{s}$, can be bounded by $c N_{\tau}$, using local $N_{\tau}$ estimates. ${ }^{9}$ Given all the machinery in Ref. 9, this is a fairly standard calculation, so it will be omitted.

\section{CONVERGENCE}

The result of "cluster expanding" (1.15), leads in analogy to (3.13) in Ref. 5 to an expansion whose coefficients have the general form ( $\Gamma$ is a set of barriers; $\partial^{\Gamma}=\Pi_{b \in \Gamma} d / d s_{b} ; \int d s_{\Gamma}$ integrates the $s_{b}$ for $b \in \Gamma$ from 0 to $\infty$ ), i.e.,

$$
\begin{aligned}
& \int d x W(x) \int d s_{\mathrm{r}} \partial^{\Gamma} T \\
& \times\left\langle\prod_{i=1}^{p} \psi \#\left(x_{i}, s\right) \exp \left[-\int H\left(\tau, s, X_{0}\right) d \tau\right]\right\rangle .
\end{aligned}
$$

where $X_{0}$ is a union of $\nabla \mathrm{s}$. It is now shown how to estimate (4.1) to obtain expressions very similar to those in Ref. 5 and thus obtain a convergence proof by the same kind of combinatorics as in Ref. 5 .

First (2.1) is used in combination with Leibniz' rule to perform $\partial^{\Gamma}$. The result is the $s$ integral of a sum of terms of the form, $y_{j} \in \mathbb{R}^{2}, y=\left(y_{j}\right)$, i. e.,

$$
\int d y K_{\boldsymbol{g}}(y, s) T\left\langle\prod_{j=1}^{m} \psi \#\left(y_{j}, s\right) \exp \left[-\int H\left(\tau, s, X_{0}\right) d \tau\right]\right\rangle,
$$

where $q$ indexes each term in the sum. Each field $\psi \#$ in (4.2) will be assumed to be localized by the kernel $K g$ in a space-time region $\nabla$, so $g$ not only indexes the ways in which the differentiations are applied (terms resulting from Leibniz' rule), but also the possible localizations of the fields.

(4.2) is estimated by taking the operator norm over the fermion Fock space in the following manner: Introduce an orthonormal product basis, $f_{\alpha}$ of $L^{2}(\mathbb{R}) \times \cdots \times L^{2}(\mathbb{R})$, with $m$ factors. Write $K \boldsymbol{g}(s)$ as a sum of $f_{\alpha}$ with coefficients that depend on $s$ and the time variables in $y$. Estimate $(4.2)$ by applying $\|\psi \#(f)\| \leqslant\|f\|_{2}$ to this sum together with the lower bound on the exponent, (3.1). Let $t$ be the multivariable $t_{1}, \ldots, t_{m}$, the times in $y .(4.2)$ is less than, in absolute value,

$$
\left.\int d t\|K g(t, s)\|_{\mathrm{D}} \exp \text { (const }\left|X_{0}\right|\right),
$$

where the (defermiation) norm \|\|$_{D}$ is defined on a function $F$ of $m$ space variables $x_{1}^{\prime}, \ldots, x_{m}^{\prime}$ by the prescription (sum over norms of components if $F$ is a matrix)

$$
\|F\|_{D}=\inf \sum_{\alpha}\left|\left\langle F, f_{\alpha}\right\rangle\right|,
$$

where the infimum is over product bases of $\Pi L^{2}(\mathbb{R})$ as above.

In the special case where $W$ is constant and there is no $\int d x$ in (4.1), the quantity $K g$ factors, together with the integrals $\int d t$, into terms $K g_{c}$, corresponding to the way in which any Feynman graph for this theory fac- tors, into its connected subgraphs which are either closed loops or lines with open ends [an open end corresponding to a field $\psi$ in (4.2)]. Because the $f_{\alpha}$ in (4.4) are product bases, the \|\|$_{D}$ norm in (4.3) also factors. A factor $K_{g}$ corresponding to a graph consisting of a connected line with $n$ vertices and both ends open has the form

$$
\begin{aligned}
& K_{g_{c}}\left(x_{1}, x_{n}, s\right) \\
& =\int d x_{2} \cdots d x_{n-1}\left(\phi \chi_{\nabla_{1}}\right)\left(x_{1}\right) S_{\gamma_{1}}\left(x_{1}, x_{2}\right)\left(\phi \chi_{\nabla_{2}}\right)\left(x_{2}\right) \\
& \quad \times S_{\gamma_{2}}\left(x_{2}, x_{3}\right)\left(\phi \chi_{\nabla_{3}}\right)\left(x_{3}\right) \cdots S_{\gamma_{n-1}}\left(x_{n-1}, x_{n}\right)\left(\phi \chi_{\nabla_{n}}\right)\left(x_{n}\right),(4.5)
\end{aligned}
$$

where $\gamma_{1}, \ldots, \gamma_{n}$ are disjoint subsets of $\Gamma$ and $S_{\gamma}$ $=\Pi_{b \in \mathrm{r}} d / d s_{b} S . x_{i} \in \mathbb{R}^{2}$. By making a specific choice of product basis $f_{\alpha}$ in (4.4), it is easy to see that, letting $x_{i}=\left(x_{i}^{\prime}, t_{i}\right)$,

$$
\left\|K_{g_{c}}\left(t_{1}, t_{n}\right)\right\|_{D} \leqslant \text { const } \operatorname{tr}\left|K_{g_{c}}\left(t_{1}, t_{n}\right)\right|,
$$

where $\left|K_{g_{f}}\left(t_{1}, t_{n}\right)\right|$ means the operator absolute value, i. e., $\left[K_{g_{c}}{ }^{k}\left(t_{1}, t_{n}\right) K_{g_{c}}\left(t_{1}, t_{n}\right)\right]^{1 / 2}$, where $K_{g_{c}}\left(t_{1}, t_{n}\right)$ denotes the operator corresponding to the kernel $K_{g_{c}}\left(x_{1}^{\prime}, t_{1}, x_{n}^{\prime}, t_{n}\right)$ for fixed $t_{1}, t_{n}$. Except when $n=2$ the trace norm in $(4,6)$ may be estimated by first taking the time integrals in (4.5) outside the trace norm and then majorizing by a product of Hilbert Schmidt norms of the form, $i=1,2, \ldots, n-1$,

$$
\begin{aligned}
\sum_{\alpha, \beta}[ & \int d x_{i}^{\prime} d x_{i+1}^{\prime} \mid\left(|\phi|^{1 / 2} \chi_{\nabla_{i}}\right)\left(x_{i}\right) \\
& \left.\times\left. S_{\gamma_{i}}^{(\alpha, \beta)}\left(x_{i}, x_{i+1}\right)\left(|\phi|^{1 / 2} \chi_{\nabla_{i+1}}\right)\left(x_{i+1}\right)\right|^{2}\right]^{1 / 2},
\end{aligned}
$$

where $\alpha, \beta$, are spinor indices. By the Hơlder inequality $(4.7)$ is less than

$\left\|\phi \chi_{\nabla_{i}}\left(t_{i}\right)\right\|_{3}^{1 / 2}\left\|S_{\gamma_{i}}\left(t_{i}, t_{i+1}\right)\right\|_{\hat{3}_{\nabla_{i}} \times \nabla_{i+1}}\left\|\phi \chi_{\nabla_{i+1}}\left(t_{i+1}\right)\right\|_{3}^{1 / 2}$,

where the \|\|$_{3, \nabla \times \nabla^{\prime}}$ norm is defined for a function $F$ $=F(x, y)$ with $x=\left(x^{p}, t\right), y=\left(y^{\prime}, u\right)$, to be the $L^{3}$ norm with respect to $x^{\prime}, y^{\prime}$ of $\chi_{\nabla}(x) F(x, y) \chi_{\nabla^{\prime}}(y)$. When $F$ is a matrix, the norm is defined to be the sum over the norms of the components. $\left\|\phi \chi_{\nabla}\right\|_{3}$ is the $L^{3}$ norm of $\phi \chi_{\nabla}$ with respect to the spatial variable. So by the hypothesis (1.13), for $n \geqslant 2$,

$$
\begin{gathered}
\int d t_{1} d t_{n}\left\|K_{q_{c}}\left(t_{1}, t_{n}\right)\right\|_{D} \leqslant(\text { const })^{n} \int d t_{1} \circ \cdot d t_{n} \\
\times \prod_{i=1}^{n-1}\left\|S_{\gamma_{i}}\left(t_{i}, t_{i+1}\right)\right\|_{3, \nabla_{i} \times \nabla_{i+1}} .
\end{gathered}
$$

The time integral is estimated by the Cauchy Schwarz inequality to obtain

$$
\int d t_{1} d t_{n}\left\|K_{g_{c}}\right\|_{D} \leqslant(\text { const })^{n} \prod_{i=1}^{n-1}\left\|S_{\gamma_{i}}\right\|_{3, \nabla_{i} \times \nabla_{i+1}} \text {, }
$$

where

$$
\begin{aligned}
& \left\|S_{\gamma_{i}}\right\|_{3, \nabla_{i} \times \nabla_{i+1,2}} \\
& \quad=\left(\int d t_{i} d t_{i+1}\left\|S_{\gamma_{i}}\left(t_{i}, t_{i+1}\right)\right\|_{3, \nabla_{i} \times \nabla_{i+1}}^{2}\right)^{1 / 2} .
\end{aligned}
$$

Suppose now that $n=2$ in (4.5). In the next section, identities and estimates for $S_{\gamma}$ are developed and with 
their aid it is a simple matter to prove directly that for some $q>0$,

$$
\begin{aligned}
& \int d s_{\gamma_{1}} \int d t_{1} d l_{2} \operatorname{tr}\left|K_{g_{c}}\left(t_{1}, t_{2}\right)\right| \\
& \quad \leq \text { const } M_{0}^{-\left|\gamma_{1}\right| / \alpha} \exp \left[-M_{0} d\left(j, \gamma_{1}\right) / 2\right],
\end{aligned}
$$

where $\int d s_{\gamma_{1}}$ integrates only over those $s_{b}$ such that $b \in \gamma_{1}$. The estimate is uniform in the remaining $s_{b}$. $d(j, \gamma)$ is defined for a localization $j$ and a line $\gamma$ to be the distance between $\nabla_{1}$ and $\nabla_{2}$ measured by the shortest path which touches every barrier $b \in \gamma$ where $\nabla_{1}$ and $\nabla_{2}$ are the localizations specified by $j$ for the variables in $S_{y}$.

In general, $K g$ also contains numerical factors corresponding to closed loops. These already have the form of a trace of an operator, hence in absolute value are less than the trace norm of that operator. The operator has a form similar to (4.5) and can be bounded by the same methods, even when $n=2$ since there is an extra propagator in the operator. Also in the next section, it will be proved that, letting $j(\gamma)$ denote the localization specified for $S_{\gamma}$,

$$
\begin{aligned}
& \int d s_{\gamma}\left\|S_{\gamma}\right\|_{\beta_{\rho} j(\gamma), 2} \\
& \quad \leqslant \text { const } M_{0}^{-|\gamma| / q} \exp \left[-M_{0} d(j, \gamma) / 2\right] .
\end{aligned}
$$

Putting all this together and using a simple argument to include $W$ gives

$$
\begin{aligned}
\mid \int d x & W(x) \int d s_{\Gamma} \partial T\left\langle\prod_{i=1} \psi \#\left(x_{i}\right)\right. \\
& \times \exp \left[-\int H\left(\tau, s, X_{0}\right) d \tau|\rangle \mid\right. \\
\leqslant & \|W\|_{2} \exp \left(\text { const }\left|X_{0}\right|\right) \\
& \times \sum_{\mathcal{g}}\left(\text { const) }{ }^{n}(\boldsymbol{\gamma}) \prod_{\gamma} M_{0}^{-|\gamma| / a} \exp \left[-M_{0} d(j, \gamma) / 2\right] .\right.
\end{aligned}
$$

The $\|W\|_{2}$ is with respect to space and time variables. This estimate is almost the same (a different localization) as estimates in Ref. 5 and leads to a proof of convergence for $M_{0}$ sufficiently large via the same combinatorics as in Ref. 3. In particular Proposition 5.3 of Ref. 5 is a consequence.

\section{ESTIMATES ON THE PROPAGATOR}

The objective is to prove (4.14). A proof of (4.13) can easily be constructed using the same techniques.

To begin with, write

$$
\exp [-|t-u| \omega(s)]=\exp \left[-|t-u| \omega_{D}(s)\right]-E,
$$

where $t, u$ are assumed to be in the same interval $J$, so that $\omega(s)=\omega(s, \tau)$ is constant for $\tau \in[t, u], \omega_{\mathrm{D}}(s)$ is defined to be the result of replacing $\Delta$ by $\Delta_{D}$ in (1.3). $\Delta_{D}$ is the Laplacian with Dirichlet conditions at every boundary point of intervals $I$, in the partition of the spatial axis. (see Sec. 1.) Now it will be shown, by constructing an integral representation for $E$, that

$$
\begin{aligned}
& \int d s_{\gamma}|| E_{\gamma} \|_{3, \nabla_{x} \times \nabla_{y_{2} 2}} \\
& \quad \leqslant \text { const } M_{0}{ }^{-|\gamma| / q} \exp \left(-M_{0} d\left(\nabla_{x}, \nabla_{y}, \gamma\right) / 2\right),
\end{aligned}
$$

where $E_{\gamma}=\partial^{\gamma} E(x, y)$ with $x=\left(x^{\prime}, t\right), y=\left(y^{\prime}, u\right) . \nabla_{x}$, the localization of $x$, is $I_{x} X J$ and $\nabla_{y}=I_{y} X J$.

The representation for $E$, by (1.10), is

$$
E(x, y)=(i \pi)^{-1} \int_{-\infty}^{\infty} d k_{0} k_{0} \exp \left(i k_{0}|t-u|\right) g\left(x^{\prime}, y^{\prime}\right),
$$

where $g\left(x^{\prime}, y^{\prime}\right)$ is the difference between two Green's functions with different boundary conditions for the differential operator $\omega^{2}(s)$. Thus, it satisfies the homogeneous equation (acting on $x^{\prime}$ )

$$
\begin{aligned}
& \left(\omega^{2}+k_{0}^{2}\right) g\left(x^{\prime}, y^{\prime}\right)=0, \\
& g\left(x^{\prime}, y^{\prime}\right)=\left(k_{0}^{2}+\omega^{2}\right)^{-1}\left(x^{\prime}, y^{\prime}\right), \text { for } x^{\prime} \in \partial I_{x},
\end{aligned}
$$

where the $s$ dependences have been suppressed. $\left(k_{0}^{2}+\omega^{2}\right)^{-1}\left(x^{\prime}, y^{\prime}\right)$ denotes the kernel of $\left(k_{0}^{2}+\omega^{2}\right)^{-1}$. Let $l\left(x^{\prime}\right)$ be the linear function of $x^{\prime}$ which coincides with $\left(k_{0}^{2}+\omega^{2}\right)^{-1}\left(x^{\prime}, y^{\prime}\right)$ when $x^{\prime} \in \partial Y_{x}$. By obtaining the equation for $g\left(x^{\prime}, y^{\prime}\right)-l\left(x^{\prime}\right)$ as a function of $x^{\prime}$, it can be seen that

$$
g\left(x^{\prime}, y^{\prime}\right)=-\left(\Delta_{\mathrm{D}}\left(k_{0}^{2}+\omega^{2}\right)^{-1} l\right)\left(x^{\prime}, y^{\prime}\right), \quad \text { for } x^{\prime} \in \partial I_{x},
$$

where the operators act on the $x^{\prime}$ variable. Let $l_{x_{-}}\left(x^{\prime}\right)$ be the linear function that equals one at $x_{-}^{\prime}$, the lefthand boundary of $I_{x}$, and equals zero at $x_{+}^{\prime}$ the right end point. Set $l_{x_{+}}=1-l_{x_{-}}$. Define $g_{\xi}\left(x^{r}\right)=\left(-\Delta_{\mathrm{D}}\left[k_{0}^{2}+\omega_{\mathrm{D}}^{2}\right]^{-1} l_{\xi}\right)$ $\left(x^{\prime}\right)$, where $\xi=x_{+}^{\prime}$ or $x_{-}^{\prime}$. Then

$$
g\left(x^{i}, y^{\prime}\right)=\sum_{\xi=x_{+1}^{\prime} x_{-}^{\prime}}\left(k_{0}^{2}+\omega^{2}\right)^{-1}\left(\xi, y^{\prime}\right) g_{\xi}\left(x^{\prime}\right) .
$$

By the same argument applied to $y^{\prime}$,

$$
\begin{aligned}
\left(k_{0}^{2}+\right. & \left.\omega^{2}\right)^{-1}\left(\xi, y^{\prime}\right) \\
& =\left(k_{0}^{2}+\omega^{2}\right)^{-1}\left(\xi, y^{\prime}\right)-\left(k_{0}^{2}+\omega_{D}^{2}\right)^{-1}\left(\xi, y^{\prime}\right) \\
& =g\left(\xi, y^{\prime}\right) \\
& =\sum_{\zeta=y_{+}^{\prime}, y_{-}^{\prime}}\left(k_{0}^{2}+\omega^{2}\right)^{-1}(\xi, \zeta) g_{\zeta}\left(y^{\prime}\right) .
\end{aligned}
$$

Combining (5.6) and (5.7),

$$
g\left(x^{\prime}, y^{\prime}\right)=\sum_{\xi, \xi}\left(h_{0}^{2}+\omega^{2}\right)^{-1}(\xi, \zeta) g_{\xi}\left(x^{\prime}\right) g_{\zeta}\left(y^{\prime}\right) .
$$

Now use the identity $\beta^{-1}=\int_{0}^{\infty} \exp (-\sigma \beta) d \sigma$ to convert resolvents to exponentials in $(5.8)$. Let $h_{\xi}\left(x^{\prime}, \sigma\right)$ $=\left(-\Delta_{\mathrm{D}} \exp \left[-\sigma \omega_{\mathrm{D}}^{2}\right] l_{\xi}\right)\left(x^{\prime}\right)$, so that $(5.8)$ becomes

$$
\begin{aligned}
g\left(x^{\prime}, y^{\prime}\right)= & \int d \sigma_{1} d \sigma_{2} d \sigma_{3} \exp \left(-k_{0}^{2}\left[\sigma_{1}+\sigma_{2}+\sigma_{3}\right]\right) \\
& \times \sum_{\xi, \xi} \exp \left[-\sigma_{2} \omega^{2}\right](\xi, \zeta) h_{\xi}\left(x^{\prime}, \sigma_{1}\right) h_{\xi}\left(y^{\prime}, \sigma_{3}\right)
\end{aligned}
$$

Substitute (5.9) into (5.3) and evaluate the $k_{0}$ integral. Let $d \sigma$ abbreviate $d \sigma_{1} d \sigma_{2} d \sigma_{3}$ and set $\mu_{t, u}(\sigma)$ $=|l-u|^{-2} \mu\left(\sigma|u-t|^{2}\right)$ where $\mu$ was defined in Sec. 1 . Then

$$
\begin{aligned}
E(x, y)= & \sum_{\zeta, \xi} \int d \sigma \mu_{t, u}\left(\sigma_{1}+\sigma_{2}+\sigma_{3}\right) h_{\xi}\left(x^{\prime}, \sigma_{1}\right) \\
& \times \exp \left[-\sigma_{2} \omega^{2}\right](\xi, \zeta) h_{\zeta}\left(y^{\prime}, \sigma_{3}\right) .
\end{aligned}
$$

Let $s_{I}=0$ unless $I \times J$ is the support of some barrier, $b$ 
say, in which case $s_{I}=s_{b}$. The $s$ dependence can be factored out of the $h \mathrm{~s}$ by noting that $\left(\exp \left[-\sigma \omega_{\mathrm{D}}^{2}\right] l_{\xi}\right)\left(x^{\prime}\right)$ $=\exp \left[-\sigma S_{I_{x}}\right]\left\{\exp \left[-\sigma\left(M_{0}^{2}-\Delta_{D}\right)\right] l_{\xi}\right\}\left(x^{\prime}\right)$, when $x^{\prime} \in I_{x}$. Let $k_{\xi}\left(x^{\prime}, \sigma\right)=-\left\{\Delta_{\mathrm{D}} \exp \left[-\sigma\left(M_{0}^{2}-\Delta_{\mathrm{D}}\right)\right] l_{\xi}\right\}\left(x^{\prime}\right)$, then

$$
\begin{aligned}
E(x, y)= & \sum_{\xi, \zeta} \int d \sigma \mu_{t, u}\left(\sigma_{1}+\sigma+\sigma_{3}\right)\left\{\left(\exp -\sigma_{2} \omega^{2}\right)(\xi, \zeta)\right. \\
& \left.\times \exp \left[-\sigma_{1} s_{I_{x}}-\sigma_{3} s_{I_{y}}\right]\right\} k_{\xi}\left(x^{\prime}, \sigma_{1}\right) k_{\xi}\left(y^{\prime}, \sigma_{3}\right) .
\end{aligned}
$$

All the $s$ dependence is inside the curly brackets. All the $x^{\prime}$ dependence is in $k_{\xi}\left(x^{\prime}, \sigma_{1}\right)$, and the $y^{\prime}$ dependence is in $k_{\xi}\left(y^{\prime}, \sigma_{3}\right)$. (Recall $x^{\prime}, y^{\prime}$ are localized in $I_{x}, I_{y}$.) Moreover, the part in curly brackets has a path space representation because

$$
\begin{aligned}
(\exp & \left.-\sigma \omega^{2}\right)(\xi, \zeta) \\
= & \int d P_{\zeta, \zeta}^{\sigma} \exp \left(-\int_{0}^{\sigma}\left(\sum_{b} s_{b} \chi_{b}+M_{0}^{2}\right) d \sigma^{\prime}\right),
\end{aligned}
$$

where $d P_{\xi, \zeta}^{\sigma}$ is the measure of Brownian motion paths starting at $\xi$ and ending at $\zeta$ at time $\sigma$. The functions $\chi_{b}$ in (5.12) abbreviate $\chi_{b}\left(X_{\sigma}, \tau\right)$ where $X_{\sigma^{\prime}}$ is the Brownian motion and $\tau$ is any time within $[t, u]$. (The $\chi_{b}$ are constant as functions of $\tau$ within $[t, u]$.) (5.12) shows that the term in curly brackets and all its derivatives with respect to $s$ are either positive functions of $s$, or negative functions of $s$, hence

$$
\begin{aligned}
& \int d s_{\gamma}\left\|E_{\gamma}\right\|_{3, \nabla_{x} \times \nabla_{y}} \leqslant \sum_{\xi, \xi} \int d \sigma \mu_{t, u}\left(\sigma_{1}+\sigma_{2}+\sigma_{3}\right) \\
& \quad \times\left|\left\{\int d s_{\gamma} \partial^{\gamma} \exp \left(-\sigma_{1} s_{I_{x}}-\sigma_{2} \omega^{2}-\sigma_{3} s_{I_{y}}\right)(\xi, \zeta)\right\}\right| \\
& \quad \times\left\|k_{\xi}\left(\sigma_{1}\right)\right\|_{3, I_{x}}\left\|k_{\xi}\left(\sigma_{3}\right)\right\|_{3, I_{y}},
\end{aligned}
$$

where \|\|$_{3, I}$ denotes the $L^{3}(I)$ norm. By the fundamental theorem of calculus and (5.12), the term in curly brackets in (5.13) is a path integral over all paths that travel from $\xi$ to $\zeta$ in time $\sigma_{2}$ and visit all differentiated bonds on the way, which can be estimated as were the covariances in Ref. 5. The norms in $(5.13)$ are easily estimated by writing $k_{\xi}\left(\sigma_{1}\right), k_{\xi}\left(\sigma_{3}\right)$ in terms of Dirichlet eigenfunctions for the intervals $I_{x}, I_{y}$ and using the Titchmarsh theorem which says that the $L^{3}$ norm is less than the $L^{3 / 2}$ norm of the Fourier (and hence Dirichlet) transform. The final result is that the left-hand side of (5.13) is less than

$$
\text { const } M_{0}^{-(|\gamma|-2) / a} \exp \left[-M_{0} d\left(\nabla_{x}, \nabla_{y}, \gamma\right) / 2\right]|t-u|^{-\eta} \text {, }
$$

where $\eta>\frac{1}{3}$ and the constant depends on $q$ and $\eta$. Thus, choosing $\eta<\frac{1}{2}$ gives (5.2). The $|t-u|^{-n}$ comes from terms in (5.13) for which $\xi=\zeta$, and the distance between $\xi$ and $\zeta$ via bonds in $\gamma$ is zero. This concludes the proof of $(5.2)$.

The next step is to incorporate the spinors to obtain, together with an analogous estimate involving $W_{+}$, i. e.,

$$
\begin{aligned}
& \int d s_{\gamma}\left\|\left(\partial^{\gamma} 1 \bar{w}_{-}\right) E_{\gamma_{2}}\left(\partial^{\gamma} w_{-}\right)\right\|_{3, \nabla_{x} \times \nabla_{y}, 2} \\
& \quad \leqslant \text { const } M_{0}^{-|\gamma| / q} \exp \left[-M_{0} d\left(\nabla_{x}, \nabla_{y}, \gamma\right) / 2\right],
\end{aligned}
$$

where $\gamma_{1} \cup \gamma_{2} \cup \gamma_{3}=\gamma$ with $\gamma_{1}, \gamma_{2}, \gamma_{3}$ disjoint and $\partial^{\gamma} \bar{w}_{-}$, $E_{\gamma_{2}}, \partial^{\gamma_{3}} w_{\text {- }}$ are being used to denote both the kernels given by $\partial^{\gamma_{1}} w_{-}, E_{\gamma_{2}}, \partial^{\gamma_{3}} w_{-}$as functions of the spatial variables, while $s, t, u$ are fixed, and the corresponding operators. (5.15) is proved with the aid of the following lemma.

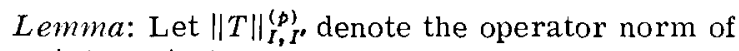
$T: L^{p}(I) \rightarrow L^{p}\left(I^{\prime}\right)$. If $T$ has spinor indices, \|\| includes a sum over the norms of components. Then,

$$
\begin{aligned}
& \int d s_{\gamma}\left\|\partial^{\gamma} w_{-}\right\|_{I, I^{\prime}}^{(p)} \\
& \quad \leq \mathrm{const} \exp \left[-M_{0}^{\prime} d\left(\nabla, \nabla^{\prime}, \gamma\right)\right],
\end{aligned}
$$

where the constant depends only on $p(1<p<\infty)$ and $M_{0}^{\prime} \leqslant \frac{9}{10} M_{0} ; \nabla=I \times J, \nabla^{\prime}=I^{\prime} \times J$. A similar lemma holds for $\bar{w}_{-}, w_{+}, \bar{w}_{+} \cdot(5.16)$ is uniform in all $s_{b}$ not integrated out, $t, u$ and $\epsilon$.

Proof: $\partial^{\gamma} u_{-}$, when restricted to $I \times I^{\prime}$ becomes the function $\partial^{\gamma} f(s)$ (see Sec. 1) times the operator of convolution by $\left.\hat{\kappa}_{\epsilon} \omega^{-1 / 2} v\right)^{\nu}$. The $\partial^{\gamma} f(s)$ can be taken outside the norm and $\int d s_{\gamma}$ evaluated by the fundamental theorem of calculus because $\left|\partial^{\gamma} f(s)\right|= \pm \partial^{\gamma} f(s)$. $\partial^{\gamma} f(s)$ vanishes if the distance between $I$ and $I^{\prime}$ is less than $d\left(\nabla, \nabla^{\prime}, \gamma\right)$; therefore, without loss of generality, assume the distance between $I$ and $I^{\prime}$ is greater than $d\left(\nabla, \nabla^{\prime}, \gamma\right) . \omega^{-1 / 2} v$ is analytic in $p$ space and the nearest singularity to the real axis is at distance $M_{0}$, hence, by a distortion of the contour in evaluating the Fourier transform, it follows that $\left(\omega^{-1 / 2} v\right)^{\bullet}(\xi)$ decays as $\exp \left(-M_{0}^{\prime}|\xi|\right)$ and is $C^{\infty}$ away from $\xi=0$. (5.16) follows except when $d\left(\nabla, \nabla^{\prime}, \gamma\right)=0$, i. e., $\nabla$ and $\nabla^{\prime}$ are contiguous. This case is easily completed by using Mihlin's theorem (Ref. 10, p. 120) to show that as a convolution operator the Fourier transform of $\omega^{-1 / 2} v$ maps $L^{p}(\mathbb{R}) \rightarrow L^{p}(\mathbb{R}), 1<p<\infty$, uniformly in $M_{0}$.

(5.15) follows by writing $E_{\gamma_{2}}=\sum_{I, I^{\prime}} \chi_{I} E_{\gamma_{0}} \lambda_{I}$, in (5.15), where $\chi_{I}$ is the characteristic function of interval $I$. Then use (5.16) and control the sum over $I, I^{\prime}$ by using some of the exponential decay in (5.16). (A factor $M_{0}^{-(|\gamma|-\mathrm{t}) / \alpha}$ may be included in the right-hand side of (5.16) on replacing $M_{0}^{\prime}$ by $M_{0}^{\prime \prime}<M_{0}^{\prime}$.)

By Leibniz' rule, (5.15) implies the same estimate for the norm of $\partial^{\gamma}\left(w_{-} E w_{-}\right)$. Therefore, by (5.1), (4.14) can now be proven in the special case where $j(\gamma)$ specifies the same time-localization for the variables $x, y$, in $S_{\gamma}$ by showing that

$$
\begin{aligned}
& \int d s_{\gamma}\left\|\left(\partial^{\gamma} \bar{w}_{-}\right)\left\{\partial^{\gamma} \exp \left[-|t-u| \omega_{D}(s)\right]\right\}\left(\partial^{\gamma} w_{-}\right)\right\|_{3, \nabla_{x} \times \nabla_{y,} 2} \\
& \quad \leq \text { const } M_{0}^{-|\gamma| / q} \exp \left[-M_{0} d\left(\nabla_{x}, \nabla_{y}, \gamma\right) / 2\right]
\end{aligned}
$$

together with a similar estimate with $w_{+}$. Note that either $\gamma_{2}=\phi$ or $\left|\gamma_{2}\right|=1$.

Proof of (5.17): Use (1.11) to express the exponential in terms of $\exp \left(-\sigma \omega_{D}^{2}\right)$. As has been done above [see (5.11) and the proof of (5.16)], take the $s$ dependences outside the norm sign and evaluate the $s$ integral by the fundamental theorem of calculus. The problem then reduces to estimating

$$
\begin{gathered}
\sum_{I} \| \chi_{\boldsymbol{I}_{x}}\left(\partial^{\gamma} 1 \bar{w}_{-}(0)\right\rangle \chi_{\boldsymbol{I}} \exp \left[-\sigma\left(M_{0}^{2}-\Delta_{\mathrm{D}}\right)\right] \\
\quad \times_{\chi_{I}}\left(\partial^{\gamma_{3}} w_{-}(0)\right) \chi_{I_{I_{y}}} \|_{3}
\end{gathered}
$$


(the sum over $I$ reduces to one term if $\gamma_{2} \neq \phi$, the term where $I \times J$ is the support of the $\left.b \in \gamma_{2}\right)$, where $\partial^{\gamma_{1}} \bar{w}_{-}(0)$ denotes the operator obtained by setting $s=0$ in $\partial^{\gamma_{1}} \bar{w}_{-}(s)$, and $\chi_{I}$ is the characteristic function of interval $I$. When $I_{x} \neq I$ and $I_{y} \neq I,(5.18)$ can be estimated by writing it out in terms of the kernels of the operators and then taking absolute values of the kernels. The kernel of $\exp \sigma \Delta_{D}$ is less than the kernel of exp $\sigma \Delta$ which is well-known to be a Gaussian. The kernel of $\partial^{\gamma} \bar{w}_{-}(0)$ is $\left(\hat{\kappa}_{\epsilon} \omega^{-1 / 2} \bar{v}\right)^{\gamma}(\xi-\xi)$ when every barrier $b \in \gamma_{1}$ intersects the straight line joining $(\xi, t)$ and $(\zeta, t)$, zero otherwise. Even when $\gamma_{1}=\phi$, the singularity of $\left(\hat{\kappa}_{\epsilon} \omega^{-1 / 2} \bar{v}\right)$ is blunted because the characteristic functions in $(5.18)$ force $\xi$ and $\eta$ to lie in different squares. Hence it is permissible to take the absolute value and still get estimates uniform in $\epsilon$. Using this information it is fairly easy to complete the estimates for the terms where $I \neq I_{x}=I_{y}$.

Consider next the term (if $\neq 0$ ) in $(5.18)$ for which $I \neq I_{x}, I=I_{y}$. In this case, use $L^{p}$ continuity of $\partial^{\gamma_{3}} w_{-}(0)$ [see the proof of $(5.16)]$, and positivity to estimate by

$$
\text { const }\left\|\chi_{I_{x}}\left(\partial^{\gamma} \bar{w}_{-}(0)\right) \chi_{I_{y}} \exp \left[-\sigma\left(M_{0}^{2}-\Delta\right)\right]_{\chi_{I_{y}}}\right\|_{3} \text {, }
$$

and estimate (5.19) as in the paragraph above. The $\operatorname{term} I=I_{x} \neq I_{y}$, if $\neq 0$, is treated the same way.

The most interesting term is $I=I_{x}=I_{y}$, if there is one. Such a term would be nonvanishing only if $\gamma_{1}=\gamma_{3}=\phi$. Since every propagator is differentiated at least once, $\gamma_{2} \neq \phi$, which forces $I \times J$ to be the support of the differentiated barrier in $\gamma_{2}$. From the proof of (5.16) it can be seen that $w_{.}(0)$ is continuous from $L^{\not}(\mathbb{R})$ to $L^{\not}(I)$ uniformly in $I$ and $\epsilon$. Hence by taking $\epsilon=0$, this term can be overestimated by

$$
F(\sigma)=\text { const }\left\|\kappa_{\varepsilon} \chi_{I} \exp \left[-\sigma\left(M_{0}^{2}-\Delta\right)\right] \chi_{I} \kappa_{\epsilon}\right\|_{3} .
$$

Upon evaluating $\left\{\int_{J} d t d u\left[\int_{0}^{\infty} d \sigma \mu_{t, u}(\sigma) F(\sigma)\right]^{2}\right\}^{1 / 2}$ in order to complete the estimate, it would appear by a power counting type of argument that there is a divergence as $\epsilon \rightarrow 0$. The point is that since $I \times J$ is the support of a barrier, there is a small volume factor $\epsilon$ which controls this divergence. This concludes the proof of (5.17), and hence $(4,14)$ in this special case.

To obtain (4.14) in general, observe from (1.9) that in general $p(x, y)$ is a product of factors as on the left-hand side of (5.1) and construct decompositions analogous to $(5.1)$ and $(5.11)$. The estimate analogous to (5.17) is easier because the $t, u$ integrals are more convergent when $l, u$ are localized in different intervals.

Note added in proof: Cluster expansions for Yukawa ${ }^{2}$ have recently been established using the method of integrating out fermions by J. Magnen and R. Seneor, preprint, Centre de Physique Théorique Ecole Polytechnique and independently by A. Cooper and L. Rosen, preprint, Department of Mathematics, University of Toronto.

\section{APPENDIX: THE YUKAWA MODEL}

When $\phi$ is a boson field, there are also functional derivatives with respect to $\phi$ coming from differentia- tions of the boson measure, and the integral with respect to this measure has to be performed after removing the fermions by means of the \|\|$_{D}$ norm. The procedure given in Sec. 4 fails in two serious ways: (1) the estimate (3.1) diverges logarithmically in $\epsilon$; (2) (4.7) and (4.8) are no good because $|\phi|$ does not exist. The way around the first difficulty is to use an expansion as in Ref. 11 to lower the cutoff in the exponent. As in Ref. 11, certain divergent loops coming from the expansion have to be renormalized. To do this, a simple modification of the techniques in Sec. 5 gives control over $S(x, y, s)-S(x, y, 0)$, when $x, y \in \nabla$, and $\nabla$ is not the support of a barrier. When $\nabla$ is the support of a barrier it is not necessary to renormalize because of the small volume of the barrier.

The other difficulty (2) may be circumvented by majorizing the norm by Hilbert Schmidt norms in a different way from (4.7) so that the majority of the $\phi$ s are "padded" by propagators, for example, the Hilbert Schmidt norm of the operator whose kernel is $x_{i}=\left(x_{i}^{\prime}, t_{i}\right)$,

$$
\begin{aligned}
& \int_{\substack{x_{i} \in \nabla_{i} \\
i=2, \ldots, n-1}} d x_{2} \cdots d x_{n-1} S_{\gamma_{1}}\left(x_{1}, x_{2}\right) \phi\left(x_{2}\right) S_{\gamma_{2}}\left(x_{2}, x_{3}\right) \\
& \quad \times \phi\left(x_{3}\right) \cdots \phi\left(x_{n-1}\right) S_{\gamma_{n-1}}\left(x_{n-1}, x_{n}\right)
\end{aligned}
$$

at fixed $t_{i}, \ldots, t_{n}$, is quite respectable. It is the square root of a boson field polynomial and can easily be estimated by using a Cauchy Schwarz estimate with respect to the boson measure to remove the square root and then using Wick's theorem to evaluate the boson integral. The resulting estimate can be expressed in terms of $L^{3}$ norms of the boson and fermion propagators. There are not enough propagators to group every boson field into a kernel like (A1) but it can be arranged that the remaining ones have cutoffs so that their $L^{3}$ norms do exist. To see how this is done, see Ref. 11 where a similar problem arises. The idea is that one can contract $\psi \mathrm{S}$ (the analog to integration by parts in boson theories) after the expansion to lower the cutoff in the exponent. Any $\psi \mathrm{s}$ left are attached to vertices with a low cutoff because they arose from contractions to the exponent.

*Junior Fellow, Michigan Society of Fellows.

${ }^{1} \mathrm{P}$. Federbush, University of Michigan Preprints.

${ }^{2}$ O. McBryan, Rockefeller University Preprint

${ }^{3}$ E. Seiler, Commun. Math. Phys. 42, 163 (1975).

${ }^{4}$ E. Seiler and B. Simon, Princeton University Preprint.

${ }^{5} \mathrm{~J}$. Glimm, A. Jaffe, and T. Spencer, Constructive Quantum Field Theory, edited by G. Velo and A. Wightman, Lecture Notes in Physics Series, No. 25 (Springer Verlag, New York, 1973).

${ }^{6} \mathrm{~J}$. Glimm and A. Jaffe, in Statistical Mechanics and Quantum Field Theory, edited by $\mathrm{C}$. DeWitt and R. Stora (Gordon and Breach, New York, 1972).

7J. Glimm, Commun. Math. Phys. 5, 343 (1967); 6, 61 (1967).

${ }^{8}$ P. Federbush, J. Math. Phys. 14, 1532 (1973).

${ }^{9}$ R. Schrader, Ann. Phys. 70, 412 (1972).

${ }^{10} \mathrm{~L}$. Hormander, Act. Math. 104, $93(1960)$.

${ }^{11} \mathrm{D}$. Brydges, "Boundedness Below for Fermion Model Theories, Part II: The Linear Lower Bound, " Commun. Math. Phys. (to be published). 